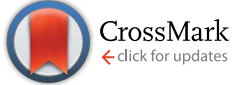

Cite this: RSC Adv., 2017, 7, 735
Received 21st October 2016 Accepted 22nd November 2016

DOI: 10.1039/c6ra25617a

www.rsc.org/advances

\section{Clean G-SERF an NMR experiment for the complete eradication of axial peaks and undesired couplings from the complex spectrum $\dagger$}

\author{
Sandeep Kumar Mishra, N. Lokesh and N. Suryaprakash* \\ The G-SERF and other related experiments are very useful in the measurement of proton-proton couplings \\ from the overlapped spectrum. However, they suffer from the presence of intense axial peaks and \\ undesirable couplings in the spectra, thereby creating ambiguity in the assignment of peaks and \\ subsequent measurement of ${ }^{1} \mathrm{H}-{ }^{1} \mathrm{H}$ couplings. In the present study we are reporting a new two \\ dimensional NMR experiment cited as Clean-G-SERF, which is able to suppress all the axial peaks and \\ unwanted couplings, while retaining only the couplings to the selectively excited proton. The method \\ permits unambiguous assignment of peaks to the coupled partners of the selectively excited proton and \\ enables the accurate measurement of couplings.
}

\section{Introduction}

The proton-proton scalar coupling information is extremely important in the conformational, stereochemical and structure elucidation of organic and bio-molecules. ${ }^{1,2}$ In small molecules, the conventional 1D ${ }^{1} \mathrm{H}$ spectrum is adequate for the measurement of ${ }^{n} J_{\mathrm{HH}}$. However, for bigger molecules the spectral complexity arises due to interaction among a large number of coupled spins, and different strengths of couplings, in addition to poorly dispersed chemical shifts. The problem gets aggravated while studying the reaction mixture due to the presence of a number of diverse molecules. Any or all such situations hinder the accurate measurement of ${ }^{n} J_{\mathrm{HH}}$ from the conventional $1 \mathrm{D}^{1} \mathrm{H}$ spectrum. Number of experiments has been reported for the precise measurement of scalar couplings, viz., $J$-upscaling in COSY spectrum ${ }^{3}$ which yields enhanced resolution and sensitivity in the indirect dimension by the cancellation of antiphase terms. Recently real time $J$-upscaling ${ }^{4}$ technique has been reported, which is applicable for the extraction of small couplings of an individual spin which experience multiple couplings, but has limitation as far as the measurement of couplings from the complex and overlapped spectra is concerned. The well-known $2 \mathrm{D}$ COSY $^{5}$ and $J$-resolved based experiments ${ }^{6}$ partially overcome such problems. However, often they fail to yield accurate coupling values, when the peaks are severely overlapped and the multiplicity pattern is complex. The 2D $\mathrm{SERF}^{7}$ (selective refocusing) experiment

NMR Research Centre, Solid State and Structural Chemistry Unit, Indian Institute of Science, Bangalore-560012, India. E-mail: nsp@sif.iisc.ernet.in; Fax: +91 80 23601550; Tel: +918022933300

† Electronic supplementary information (ESI) available. See DOI: 10.1039/c6ra25617a overcomes such a problem to a large extent by retaining only one of the desired couplings in the spectrum permitting the accurate and unambiguous assignment of peaks of the coupled partner, thereby enabling the precise measurement of coupling. Nevertheless, the SERF experiment consequent to its nature of allowing the measurement of a single coupling from each experiment requires multiple measurements demanding considerable investment of the instrument time. Gradient encoded SERF experiment (G-SERF), ${ }^{8}$ circumvents this problem as it provides access to all the couplings of a selected spin from a single experiment. Reported PCR-COSY ${ }^{9}$ for ${ }^{n} J_{\mathrm{HH}}$ measurement also suffers from the problems of resolution and sensitivity. The Push-G-SERF ${ }^{\mathbf{1 0}}$ increases the resolution of the spectrum by removing the couplings in the chemical shift dimension. The PSYCHEDELIC ${ }^{11}$ experiment wherein the data is acquired in a three dimensional fashion not only achieves the resolution by decoupling in the direct dimension but also enhances the sensitivity. The real time based one dimensional Quick-G-SERF ${ }^{12}$ technique provides information analogous to that of G-SERF, but in a much shorter time. However, this method has a drawback in the measurement of couplings of smaller magnitudes. A pure shift based $J$-edited technique provides the clean 1D spectrum but requires tedious process of data acquisition and unconventional way of processing. ${ }^{13}$ The major problem in all the slice selective NMR experiments is low sensitivity since effectively very small percentage of total proton spins are utilized in the individual slices, while the major part of total available spins is left unutilized. This problem has already been addressed ${ }^{14}$ in one dimensional and many two dimensional sequences including G-SERF. The $J$-resolved based 2D methods, such as, G-SERF, the combination of real time pure shift $^{15}$ and G-SERF known as Push-G-SERF ${ }^{10}$ and PSYCHE ${ }^{16}$ based PSYCHEDELIC ${ }^{11}$ retain more intense peaks from un- 
evolved couplings during $t_{1}$ period, known as axial peaks. These axial peaks do not give any additional information, but can merge with peaks of smaller coupling strengths, posing a challenge for the assignment of peaks and extraction of couplings. In addition, the G-SERF, PSYCHEDELIC and Push-G-SERF experiments also retain unwanted couplings that arise due to coupled protons that have close chemical shifts, which are not resolved by gradient slices and the soft pulses contained in the respective pulse sequences. Such couplings can also create a lot of confusion, in the assignment of peaks to a selected proton and the situations of this type arise more often due to small chemical shift dispersion of protons. A recent review provides the detailed account of the advantages and drawbacks of the available homonuclear broadband decoupling techniques and their applications. ${ }^{\mathbf{1 7}}$

In the present study we are introducing a novel technique cited as Clean-G-SERF (axial peaks free gradient-encoded selective refocusing) with the main focus on the eradication of such axial peaks and the peaks arising from uncoupled protons. The pulse sequence involves the manipulation of spin dynamics in such a way that the magnetization of scalar couplings from a selected proton is efficiently filtered before $t_{1}$ evolution, while the magnetization from the remaining spins is completely suppressed, which otherwise would have appeared as axial peaks. The resultant spectrum retains only the peaks from the coupled partners to the chosen proton, which significantly aids in the unambiguous assignment of peaks and the subsequent measurement of couplings even from the crowded regions of the spectrum. Another distinct advantage of the present method is the eradication of undesired couplings that evolve due to closely resonating protons that are coupled among themselves, which may or may not be coupled to the selectively excited proton, and are unresolvable both by gradient slices or soft pulses. The experiment finds utility in many chemical applications, viz., in the measurement of small couplings from the crowded spectrum of the complex molecules and in the reaction mixture containing diverse molecules where there is severe signal overlap in both the aliphatic and aromatic regions.

\section{Results and discussion}

\section{Applications}

L-Menthol. Initially the developed pulse sequence has been demonstrated on L-menthol molecule and the derived results are compared with the conventional G-SERF ${ }^{8}$ experiment (Fig. 1). The selective excitation of a particular proton without disturbing the other signals is limited by the experimental restriction of selective excitation. Thus, the selective excitation is not possible where two or more signals are overlapped and the spectrum is severely overcrowded, which is also a limitation of this technique. In the menthol molecule the proton 6e, identified by the red circle in the molecular structure (Fig. 1(a)) which gives rise to multiplet at $1.98 \mathrm{ppm}$ in the ${ }^{1} \mathrm{H}$ NMR spectrum (Fig. 1(b)), was chosen for the identification of peaks belonging to its coupled partners and the measurement of their coupling strengths.

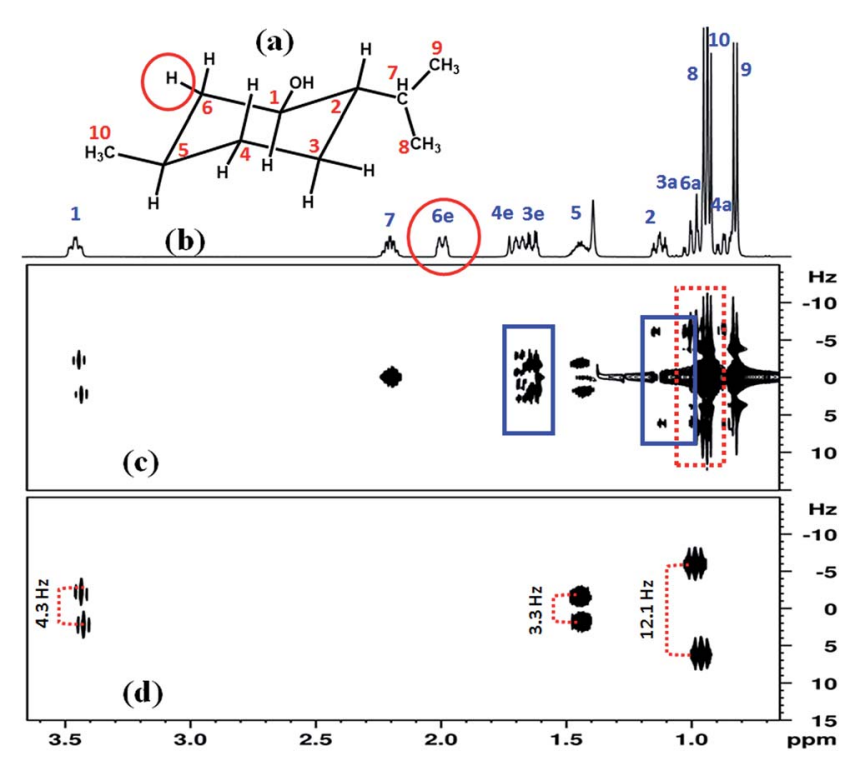

Fig. 1 (a) The chemical structure of chair form of L-menthol with the numbering of backbone; (b) the $500 \mathrm{MHz} 1 \mathrm{D}-{ }^{1} \mathrm{H}$ NMR spectrum with peak assignments; (c) the 2D G-SERF spectrum and (d) Clean-G-SERF spectrum (obtained using the present pulse sequence), of $L$-menthol in the solvent $\mathrm{CDCl}_{3}$.

It is clearly evident from the G-SERF spectrum that very intense axial peaks belonging to the uncoupled protons create confusion in the identification of peaks and the subsequent measurement of couplings. The spectrum also displays additional peaks, identified by blue rectangles (Fig. 1(c)), arising due to the small chemical shift difference between two protons coupled among themselves, but are not coupled to the selected proton. These undesirable extra peaks create ambiguity among the peaks of the uncoupled and coupled partners of the selected proton. The equatorial proton of backbone numbered 6 has three coupled partners, i.e. $6 \mathrm{a}(0.97 \mathrm{ppm}$, axial proton at position 6), proton at position $5(1.44 \mathrm{ppm})$ and proton at position 1 (3.43 ppm) marked in Fig. 1(a). From the G-SERF experiment the couplings of $6 \mathrm{e}$ with protons 1 and 5 can conveniently be measured, but the $6 \mathrm{a}$ peak is severely overlapped with the strong axial peaks of protons $3 \mathrm{a}, 8$ and 10 preventing its assignment as well as measurement of coupling [identified by dotted red rectangle]. On the other hand, the present Clean-GSERF experiment completely nullifies all the axial peaks and unwanted couplings while retaining only the peaks from the coupled partners, which is clearly evident just by the visual inspection of the spectrum (Fig. 1(d)). The measured couplings are reported in Fig. 1(d).

Strychnine. For demonstrating the wider applicability of the present technique, a complex molecule strychnine, whose structure is given in Fig. 2(a) was chosen. The proton 15b identified by a red circle in Fig. 2(a), appearing as a multiplet at 2.37 ppm (Fig. 2(b)) was chosen to identify its coupled partners and the measurement of coupling strengths associated to them.

In the G-SERF ${ }^{8}$ spectrum reported in Fig. 2(c) the coupled partner of this selected proton resonating at $3.15 \mathrm{ppm}$ exhibits severe overlap with the axial peaks from other protons, which is 


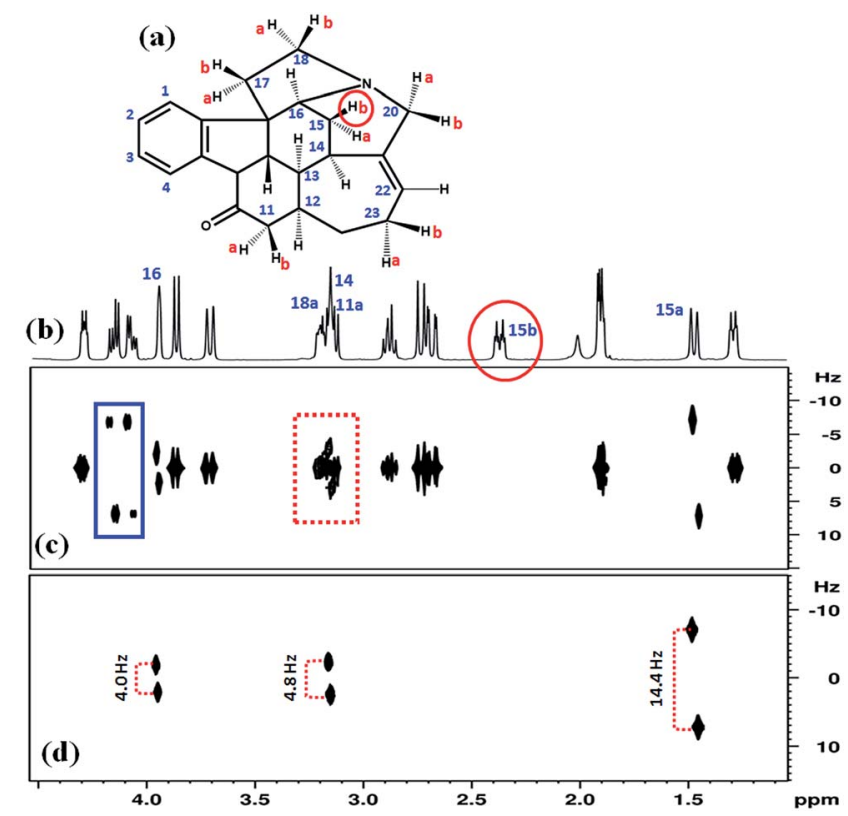

Fig. 2 (a) The chemical structure of the strychnine molecule along with the numbering of protons; (b) $1 \mathrm{D}-{ }^{1} \mathrm{H}$ NMR spectrum with the assignment of selected peaks; (c) conventional 2D G-SERF spectrum; and (d) the Clean-G-SERF spectrum of the strychnine molecule in the solvent $\mathrm{CDCl}_{3}$ acquired on a $500 \mathrm{MHz}$ NMR spectrometer. The measured couplings to proton $15 \mathrm{~b}$ have also been reported.

identified by dotted red rectangle. The unwanted peaks near 4.1-4.2 ppm identified by blue rectangle create confusion in the recognition of peaks arising due to couplings with the selected proton. These unwanted couplings are arising from the two coupled protons which are resonating very close to each other in the NMR spectrum and cannot be resolved by slice selective gradients and the used soft pulses. This type of situation is encountered very often in the proton spectrum of many organic molecules due to very small dispersion of chemical shifts. The distinct advantage of the Clean-G-SERF is clearly evident from the Fig. 2(d), where all the unwanted couplings and axial peaks are completely eradicated (Fig. 2(d)), while retaining only the couplings associated with that of selected proton $15 \mathrm{~b}$.

2-Chloro- $N^{\prime}$-(2-methoxybenzoyl)benzohydrazide and 2(diphenylphosphanyl)cyclohexan-1-amine. The present experiment has enormous utility for analysing the aliquot during the reaction monitoring. In the reaction mixture, invariably several reactants, intermediates and products can be present. In such a situation the G-SERF ${ }^{8}$ spectrum shows the strong axial peaks and also the peaks from the unwanted coupled protons that can overlap with the peaks of interest. On the other hand, the present method circumvents all these problems, which is illustrated for the molecular mixture where there is a severe overlap in the aliphatic as well as in the aromatic regions of the spectrum. The molecular structures of 2-chloro- $N^{\prime}$-(2-methoxybenzoyl)benzohydrazide and 2-(diphenylphosphanyl) cyclohexan-1-amine, and their aromatic regions of ${ }^{1} \mathrm{H}$ NMR spectrum obtained (1:1 molar ratio) in the solvent $\mathrm{CDCl}_{3}$ are reported in Fig. 3(a) and (b) respectively. The severe overlap of peaks in the region 7.3-7.5 ppm is clearly evident from the

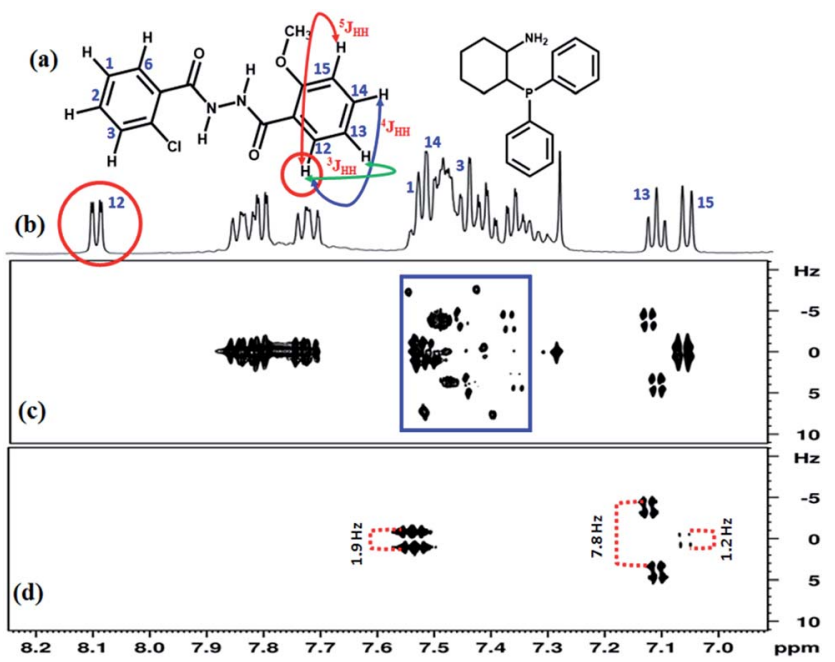

Fig. 3 (a) The chemical structure of 2-chloro- $N^{\prime}$-(2-methoxybenzoyl) benzohydrazide molecule along with the numbering of protons and the chemical structure of 2-(diphenylphosphanyl)cyclohexan-1amine; (b) few selected frequencies have been assigned in the $1 \mathrm{D}-{ }^{1} \mathrm{H}$ NMR spectrum; (c) conventional 2D G-SERF spectrum; and (d) CleanG-SERF spectrum of a mixture of 2-chloro- $N^{\prime}-(2-$ methoxybenzoyl) benzohydrazide and 2-(diphenylphospanyl)cyclohexan-1-amine molecules in the solvent $\mathrm{CDCl}_{3}$ acquired on a $500 \mathrm{MHz} N M R$ spectrometer.

Fig. 3(b). The G-SERF spectrum for this region exhibits several unwanted couplings and also axial peaks, which are identified by blue rectangle. In this spectrum it is challenging to distinguish the peaks from the coupled partners of proton 12 in 2chloro- $N^{\prime}$-(2-methoxybenzoyl)benzohydrazide. On the other hand, by applying the present technique all the unwanted peaks have been completely wiped out, providing very clean doublets from which the ${ }^{4} J_{\mathrm{HH}}$ between protons 12 and 15 of methoxy substituted aromatic ring could be precisely measured. All the three couplings, ${ }^{2} J_{\mathrm{HH}},{ }^{3} J_{\mathrm{HH}}$ and ${ }^{4} J_{\mathrm{HH}}$ with its coupled partner are identified in the Clean-G-SERF spectrum reported in Fig. 3(d). On careful inspection one can see that each peak of the doublet corresponding to proton 13 further appears as a doublet in the indirect dimension and the multiplet pattern appears like a doublet of doublet (dd). It has already been reported in our previous study that these are the peaks arising from two different conformers of 2-chloro- $N^{\prime}$-(2-methoxybenzoyl)benzohydrazide molecule in the solution. ${ }^{18}$ The coupling measured for one of the conformers is marked in Fig. 3(d) and its value is $7.8 \mathrm{~Hz}$.

3-Butyne-2-ol, progesterone and 1-pentyne-3-ol. The experiment was also demonstrated on another mixture (3-butyne-2-ol, progesterone and 1-pentyne-3-ol), whose structures are given in Fig. 4(a). All the ingredients were taken in approximately $1: 1: 1$ molar ratio. The proton 2 (identified by red circle) resonating at $4.54 \mathrm{ppm}$ in Fig. 4(b) was chosen for the extraction of couplings associated with it. The spectrum of this molecule has severe crowding of peaks in the aliphatic region. The G-SERF ${ }^{8}$ spectrum given in Fig. 4(c) reveals intense axial peaks and several unwanted couplings at 1.7 and between $2.4-2.5 \mathrm{ppm}$ which are identified by blue rectangles. The ${ }^{4} J_{\mathrm{HH}}$ between 


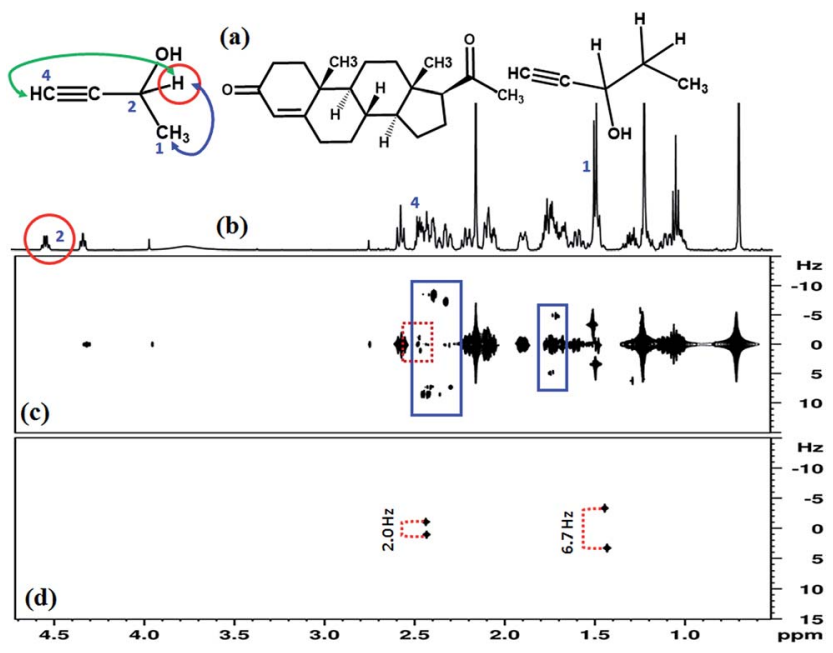

Fig. 4 (a) The chemical structures of 3-butyne-2-ol, progesterone and 1-pentyne-3-ol; (b) few peaks are assigned in the $1 \mathrm{D}-{ }^{1} \mathrm{H}$ NMR spectrum; (c) the standard 2D G-SERF spectrum; and (d) the Clean-GSERF spectrum, of 3-butyne-2-ol, progesterone and 1-pentyne-3-ol molecules in the solvent $\mathrm{CDCl}_{3}$ acquired on a $500 \mathrm{MHz} \mathrm{NMR}$ spectrometer

protons 2 and 4 is $2.0 \mathrm{~Hz}$, which is identified by a dotted red rectangle. It has appeared with other couplings and is very difficult to distinguish. On the other hand, the Clean-G-SERF spectrum reported in Fig. 4(d) contains only the peaks from the coupled partners of proton 2 . The long range couplings, ${ }^{3} J_{\mathrm{HH}}$ and ${ }^{4} J_{\mathrm{HH}}$ with their coupled partner could easily be identified and accurately measured from the Fig. 4(d). It may be pointed out that the present concept of removal of the axial peaks can also be implemented in other similar experiments, such as, Push-G-SERF, PSYCHEDELIC, etc.

The pulse sequence. The executable pulse program code for BRUKER NMR spectrometer is reported in the $\operatorname{ESI} \dagger$ and the corresponding pulse sequence is reported in Fig. 5 .

The present pulse sequence can be discussed into two parts. The first part is the excitation block (selective $90^{\circ}$ - delay $\Delta-$ hard $90^{\circ}$ pulse), and the second part is the conventional G-SERF

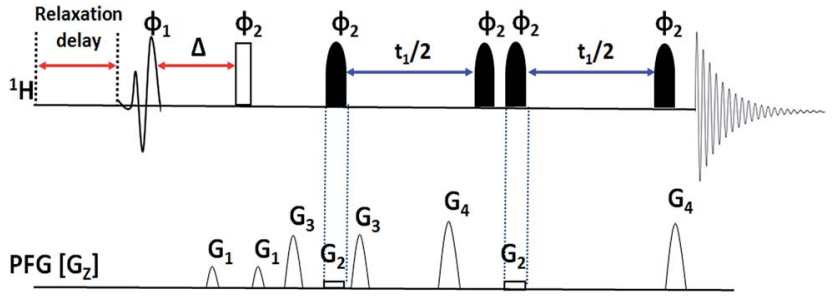

Fig. 5 Clean-G-SERF pulse sequence. On the ${ }^{1} \mathrm{H}$ channel, open shaped pulse, open rectangular pulse and closed solid shape pulse represents $\pi / 2$ selective, $\pi / 2$ hard and $\pi$ selective respectively. On the pulsed-field gradient (PFG) channel (Gz), white ellipsoids represent a shaped $z$-field gradient and white rectangles refer to slice selective $z$ gradient pulses. The length of the gradient used was $1 \mathrm{~ms}$. for all the experiments. The phase cycling employed was; $\varphi_{1}=(x,-x), \varphi_{2}=(x, x$, $-x,-x)$ and $\varphi_{\text {rec }}=(x,-x)$. The different heights of gradient pulses depict different gradient strengths. block. The initial pulse block is vital in avoiding undesired couplings and axial peaks. It is a selective COSY type sequence, which correlates selective proton magnetization with only coupled protons (similar to 1D selective COSY) which is detected and appears as the spectrum. The initial block starts with a $90^{\circ}$ selective pulse to ensure selective excitation of a proton followed by delay $(\Delta)$ and $90^{\circ}$ hard pulse to transfer magnetization selectively to the coupled protons. Thus the excitation block in Clean-G-SERF retains only desired proton unlike GSERF where all the protons are excited (desired and undesired), which is illustrated in the scheme 1.

The resultant magnetization (which is free from axial peaks and undesired couplings) is fed to the next part of the sequence (conventional G-SERF). Hence only the filtered magnetization is encoded spatially by the application of $180^{\circ}$ selective pulse with very weak field gradient field making it slice selective, which is then followed by $t_{1}$ evolution. During $t_{1}$ evolution, only the couplings from protons interacting with the selected proton are allowed to evolve by the application of $180^{\circ}$ selective pulse with very weak field gradient and another selective $180^{\circ}$ pulse on the chosen proton. The application of this $180^{\circ}$ selective pulse ensures refocusing of the chemical shift of selected proton during $t_{1}$ evolution. Since unlike G-SERF here other protons are not excited and hence no axial peaks and other undesired peaks are observed. The value of $\Delta$ should be optimized for different molecules and the optimization process is discussed in the later part of the manuscript.

The theoretical background for the initial block of the pulse sequence. The initial block of the pulse sequence is separately shown in Fig. 6. The selective soft pulse followed by the free precession of constant time period excites the bilinear terms of spin $I$ (the selected spin). The $90^{\circ}$ mixing pulse transfers the coherence to spins which are in antiphase to spin a i.e. the coupled partner of the selected spin. The density matrix for the two coupled spins evolves as the following product operators at the end of the constant delay $\Delta$.

$$
\begin{aligned}
& -I y \cos (\Omega \Delta) \cos (\pi J \Delta)+2 I x S z \cos (\Omega \Delta) \sin (\pi J \Delta) \\
& +I x \sin (\Omega \Delta) \cos (\pi J \Delta)-2 I y S z \sin (\Omega \Delta) \sin (\pi J \Delta)
\end{aligned}
$$

here $I$ and $S$ are the two coupled proton spins, $J$ is the coupling constant and $\Delta$ is the delay and the spin $I$ is selected for excitation. After next $90^{\circ} x$ pule the product operator terms end up as;

$$
-I z \cos (\Omega \Delta) \cos (\pi J \Delta)-2 I x S y \cos (\Omega \Delta) \sin (\pi J \Delta)
$$

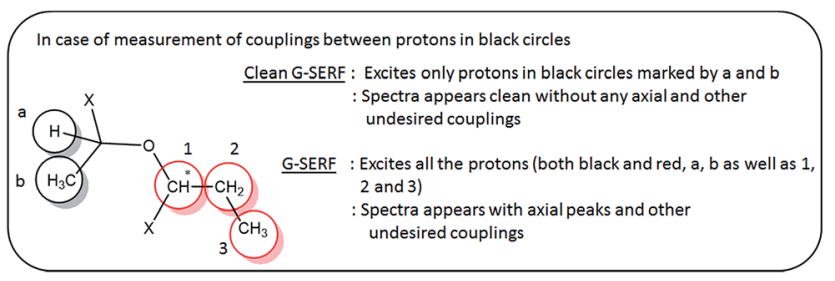

Scheme 1 Describes difference in excitation, evolution and spectra appearance between G-SERF and Clean-G-SERF experiment in chosen molecule. 


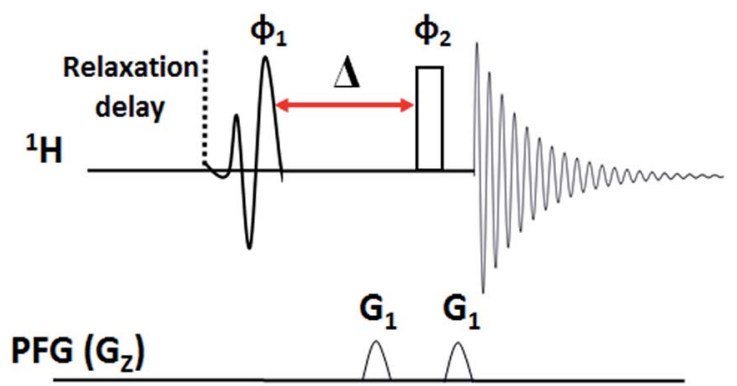

Fig. 6 The pulse sequence for optimization of $\Delta$ (optimum delay for polarization transfer). On the ${ }^{1} \mathrm{H}$ channel the first and the second pulses represents selective and hard $\pi / 2$ pulses respectively. On the pulsed-field gradient (PFG) channel (Gz), unfilled semi-ellipsoids represent a shaped $z$-field gradient. The phase cycling employed was; $\varphi_{1}=(x,-x), \varphi_{2}=(x, x,-x,-x)$ and $\varphi_{\text {rec }}=(x,-x)$.

$$
+I x \sin (\Omega \Delta) \cos (\pi J \Delta)+2 I z S y \sin (\Omega \Delta) \sin (\pi J \Delta)
$$

Eqn (1) contains the zero and double quantum terms, which are discarded by using gradients and thus we only have the resultant terms of the magnetization that is passing through the conventional G-SERF block.

$$
+I x \sin (\Omega \Delta) \cos (\pi J \Delta)+2 I z S y \sin (\Omega \Delta) \sin (\pi J \Delta)
$$

The similar terms will also be observed for the other coupled partners of the selected spin and the spins apart from these do not appear in the spectrum.

\section{Experimental}

All the investigated molecules except 2-chloro- $N^{\prime}$-(2-methoxybenzoyl)benzohydrazide were purchased from sigma Aldrich and used as received. The molecule 2-chloro- $N^{\prime}$-(2-methoxybenzoyl)benzohydrazide was synthesized in our laboratory and the procedure for synthesis has already been reported. ${ }^{18}$ Concentration of samples was approximately $15 \mathrm{mM}$ in the solvent $\mathrm{CDCl}_{3}$, for each molecule, as well as the mixture. The reported spectra from Clean-G-SERF as well as from G-SERF were obtained by keeping all the common experimental parameters identical for both the experiments. All the spectra were recorded on a $500 \mathrm{MHz}$ NMR spectrometer using a triple resonance probe with inverse $\mathrm{X}$ nuclei (TXI) at ambient temperature ( $298 \mathrm{~K})$.

The acquisition parameters used for all the individual molecules and the molecular mixtures in G-SERF and Clean-GSERF experiments are tabulated in the ESI. $\uparrow$ The spectral width in the indirect dimension for all the experiments was $80 \mathrm{~Hz}$ and the time domain points for different molecules were different. Hence the digital resolution is not identical for all the spectra. The data was processed in magnitude mode which results in some line broadening in the spectra, analogous to the G-SERF experiment.

\section{Optimization of delta $(\Delta)$}

The value of $\Delta$ can be different for different molecules and also for different protons in a molecule depending on the strengths of couplings with its partners, and hence required to be optimized before starting the actual experiment. For optimization in the present experiments, the pulse sequence given in Fig. 6 was employed. Initially the $\Delta$ value was set to $10 \mathrm{~ms}$ and varied until the signal intensities were maximum and comparable. The number of dummy scans was set to 2 and total number of accumulations was set to 2 . The homospoil $z$ only gradient; $G_{1}=$ $15 \%$ was used. The selective $\pi / 2$ pulse for excitation was EBurp, and the same pulse length was used in the Clean-G-SERF experiment.

The Fig. 7 represents the stack plot of different $1 \mathrm{D}^{1} \mathrm{H}$ spectra of the molecule strychnine acquired using pulse sequence given in Fig. 6. The intensities of the coupled partners of selected proton vary on altering the delay $\Delta$. The optimum value of $\Delta$ for the Clean-G-SERF experiment was chosen when the signal intensities for all the coupled partners were maximum and nearly equal. From Fig. 7 one can clearly see that for $\Delta$ value of $32 \mathrm{~ms}$ the signal intensities at $3.2 \mathrm{ppm}$ and $4 \mathrm{ppm}$ are very less and on increasing the value to $70 \mathrm{~ms}$ the signal of proton resonating at $1.5 \mathrm{ppm}$ almost disappeared. At $52 \mathrm{~ms}$ signal intensities are comparable and hence was chosen as the optimum value for this molecule. The similar procedure is required to be followed for optimizing the delay for other molecules.

The optimization of $\Delta$ is also advantageous in certain situations where the intensity difference between two peaks is considerably large. In such a situation, the value of $\Delta$ can be optimized to selectively manipulate the intensity of a particular peak.

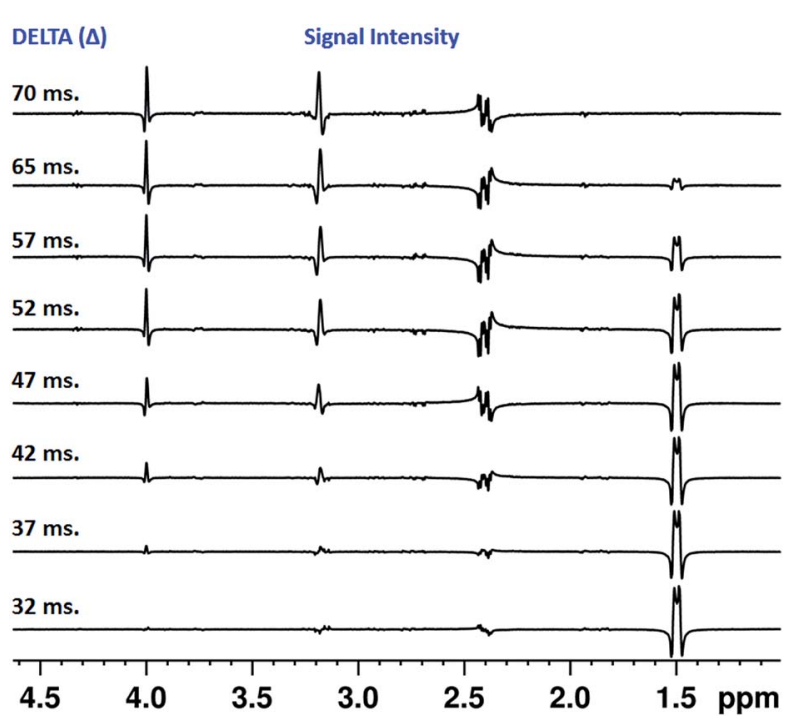

Fig. 7 The stack plot showing the effect of different values of $\Delta$ on the signal intensities of coupled partners of the selected proton. The peak near 2.4 ppm was chosen for selective excitation. Selective $\pi / 2$ pulse EBurp (40 ms) with $112.8 \mathrm{~Hz}$ excitation bandwidth was used. All these $1 \mathrm{D}{ }^{1} \mathrm{H}$ spectra were acquired on a $400 \mathrm{MHz}$ NMR spectrometer. 


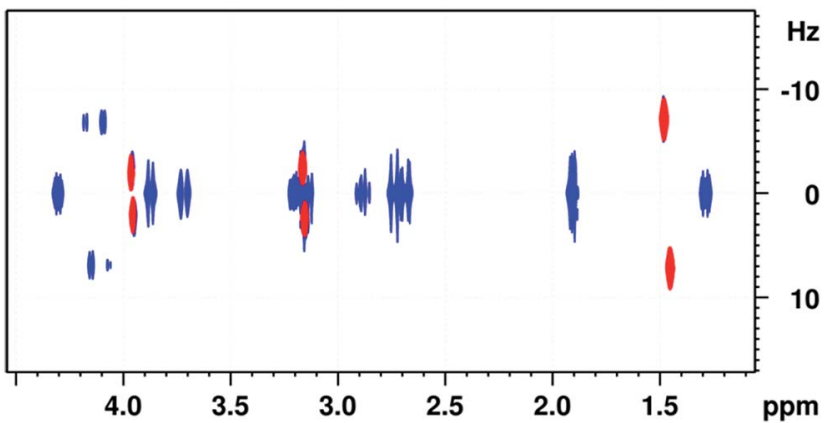

Fig. 8 The superimposed spectra of strychnine molecule obtained from the G-SERF (blue peaks with axial peaks and false couplings in the spectrum), and Clean-G-SERF (red contour with no extra peaks in the spectrum other than the coupled partners of selected proton) techniques. Both the spectra are acquired on a $500 \mathrm{MHz}$ NMR spectrometer at $298 \mathrm{~K}$ and plotted with equal intensity scale.

\section{Sensitivity of G-SERF vs. Clean-G-SERF}

To compare the sensitivity of the present experiment with GSERF $^{8}$ sequence we have carried out both the experiments with the identical common parameters and the obtained integral values of the peaks from the G-SERF and Clean-G-SERF are given in ESI (the Tables $2 S$ and $3 S \dagger$ respectively). The obtained ratio of absolute integrals for G-SERF/Clean-G-SERF for the peaks at $1.49,3.17$ and $3.96 \mathrm{ppm}$ are $0.5786,0.6274$ and 0.7498 respectively. On the other hand, when we compare the signal to noise ratio of G-SERF/Clean-G-SERF, these values for peaks at $1.49,3.17$ and 3.96 are $1.40,1.52$ and 1.82 respectively. For visual comparison the superimposed 2D spectra obtained from both G-SERF and Clean-G-SERF is given in Fig. 8.

\section{Conclusions}

The Clean-G-SERF experiment offers unique solution for the identification of peaks from the coupled partners of a selected proton thereby enabling the measurement of homonuclear couplings in diverse situations. Its superiority over similar experiments in efficient suppression of axial peaks and the unwanted couplings has been convincingly demonstrated. The application of the sequence for deriving the strengths of small couplings from a complex spectrum and from the severely overlapped peaks from the molecular mixtures containing different components has been established. The pulse sequence is simple and the experiment is easy to implement, in addition to providing excellent spectral quality. We have a strong feeling that this experiment might find utility in diverse situations, where the accurate measurement of scalar or residual dipolar couplings is a requirement. It may be pointed out that the smallest coupling measurable from the present experiment, depends on the achievable resolution in the indirect dimension. In the present study, the smallest measured coupling is $1.2 \mathrm{~Hz}$. The present experiment might also find utility in structural, configurational and conformational analysis of natural products, intermediate and products of reaction mixture and the individual or mixture of complex organic molecules.

\section{Acknowledgements}

SKM would like to thank UGC, New Delhi for Senior Research Fellowship. NS gratefully acknowledges the generous financial support by the Science and Engineering Research Board (SERB), New Delhi (Grant Number: EMR/2015/002263).

\section{Notes and references}

1 (a) M. Eberstadt, G. Gemmecker, D. F. Mierke and H. Kessler, Angew. Chem., Int. Ed., 1995, 34, 1671; Angew. Chem., 1995, 107, 1813; (b) M. Karplus, J. Am. Chem. Soc., 1963, 85, 2870; M. Karplus, J. Chem. Phys., 1959, 30, 11. (c) Y. EnomotoRogers, H. Masaki, I. Tetsuya, F. Kazuo and I. Tadahisa, Magn. Reson. Chem., 2016, 54, 561.

2 (a) G. Bifulco, P. Dambruoso, L. Gomez-Paloma and R. Riccio, Chem. Rev., 2007, 107, 3744; (b) R. H. Contreras and J. E. Peralta, Prog. Nucl. Magn. Reson. Spectrosc., 2000, 37, 321; (c) W. A. Thomas, Prog. Nucl. Magn. Reson. Spectrosc., 1997, 30, 183.

3 (a) L. R. Brown, J. Mag Res., 1984, 57, 513; J. Mag. Res., 1984, 57, 513; (b) R. V. Hosur, K. V. R. Chary and M. Ravi Kumar, Chem. Phys. Lett., 1985, 116, 105.

4 S. Glanzer and K. Zangger, J. Am. Chem. Soc., 2015, 137, 5163. 5 (a) C. Griesinger, O. W. Sørensen and R. R. Ernst, J. Am. Chem. Soc., 1985, 107, 6394; (b) C. Griesinger, O. W. Sørensen and R. R. Ernst, J. Chem. Phys., 1986, 85, 6837; (c) R. Brischweiler, J. C. Madsen, C. Griesinger, O. W. Sørensen and R. R. Ernst, J. Magn. Reson., 1987, 73, 380.

6 (a) W. P. Aue, J. Karhan and R. R. Ernst, J. Chem. Phys., 1976, 64, 4226; (b) D. Marion and K. Wüthrich, Biochem. Biophys. Res. Commun., 1983, 113, 967; (c) M. Ottiger, F. Delaglio and A. Bax, J. Magn. Reson., 1998, 131, 373; (d) B. L. Marquez, W. H. Gerwick and R. T. Williamson, Magn. Reson. Chem., 2001, 39, 499.

7 T. Facke and S. Berger, J. Magn. Reson., Ser. A, 1995, 113, 114. 8 N. Giraud, L. Béguin, J. Courtieu and D. Merlet, Angew. Chem., Int. Ed., 2010, 49, 3481; Angew. Chem., 2010, 122, 3559.

9 N. Giraud, D. Pitoux, J. M. Ouvrard and D. Merlet, Chem.Eur. J., 2013, 19, 12221.

10 D. Pitoux, B. Plainchont, D. Merlet, Z. Y. Hu, D. Bonnaffe, J. Farjon and N. Giraud, Chem.-Eur. J., 2015, 21, 9044.

11 D. Sinnaeve, M. Foroozandeh, N. Mathias and G. A. Morris, Angew. Chem., Int. Ed., 2016, 55, 1090.

12 (a) N. Lokesh, S. R. Chaudhari and N. Suryaprakash, Chem. Commun., 2014, 50, 15597; (b) N. Gubensäk, W. M. F. Fabian and K. Zangger, Chem. Commun., 2014, 50, 12254.

13 S. R. Chaudhari and N. Suryaprakash, ChemPhysChem, 2015, 16, 1079.

14 Lokesh and N. Suryaprakash, Chem. Commun., 2014, 50, 8550-8553.

15 N. H. Meyer and K. Zangger, Angew. Chem., Int. Ed., 2013, 52, 7143. 
16 M. Foroozandeh, R. W. Adams, N. J. Meharry, D. Jeannerat, 17 L. Castañar and T. Parella, Magn. Reson. Chem., 2015, 53, N. Mathias and G. A. Morris, Angew. Chem., Int. Ed., 2014, 53, 6990. 399.

18 S. K. Mishra and N. Suryaprakash, Phys. Chem. Chem. Phys., 2015, 17, 15226. 